\section{Clinical leishmaniasis in dogs living in UK}

\section{Paolo Silvestrini ${ }^{1,8}$, Dan Batchelor ${ }^{1}$, Alex German', Christina Maunder², Tracy Hill ${ }^{3}$, Goncalo Serrano4, Karin Allenspach ${ }^{5}$, Aran $\mathrm{Mas}^{6}$, Mayank Seth ${ }^{7}$, Marta Planellas ${ }^{8}$, Josep Pastor ${ }^{8}$, Xavier Roura ${ }^{8}$}

\author{
University of Liverpool, Liverpool, UK \\ University of Bristol, Bristol, UK \\ University of Edinburgh, Edinburgh, UK \\ University of Cambridge, Cambridge, UK \\ Royal Veterinary College, London, UK \\ Anderson and Moores Veterinary Specialists, Winchester, UK \\ Animal Health Trust, Newmarket, UK \\ Autonomous University of Barcelona, Barcelona, Spain
}

Canine leishmaniasis (CanL) is spreading to non-endemic areas with an increasing number of cases reported in Northern Europe. In the UK, where no natural transmission occurs, it has become an important infectious disease in dogs with a history of travel. Given that there are no pathognomonic signs, CanL is easily misdiagnosed with other infectious or immune-mediated diseases. The objective of this retrospective multi-centre study was to describe the different aspects of CanL in dogs living in UK.

Seven veterinary referral centres in the UK were contacted and asked to review their clinical records for confirmed cases of CanL. A total of thirty-seven dogs were finally included. The majority were of mixed breed (32\%) with a median age of 4.8 years (range 1.11 years-12.2 years). All dogs had a history of adoption from or travel to endemic areas, especially Spain. No autochthonous cases were recognised. Diagnosis was reached by serology (ELISA or IFAT), PCR and cytology. PCR was performed on blood, bone marrow, spleen, lymph nodes, skin, conjunctiva and joint fluid. Leishmania amastigotes were detected by cytology of lymph nodes, spleen, bone marrow and skin. The most frequent causes of presentation were lethargy $(20 / 37,54 \%)$, dermatologic problems (16/37, 43\%) and lameness (8/37,22\%). Systemic lymphadenopathy (21/37, $57 \%)$, multifocal alopecia and crusting $(17 / 37,46 \%)$ and polyarthritis $(9 / 37,24 \%)$ were the most common clinical findings. Anaemia was present in 19/31 (61\%) dogs and was most frequently non-regenerative (67\%). Eight of 22 (36\%) were thrombocytopenic and 2/21 (9\%) pancytopenic. Renal azotaemia was found in 6/24 (25\%) patients and proteinuria in 19/26 (73\%). Twenty-seven of 29 (93\%) animals were hypoalbuminaemic and hyperglobulinaemic. Thirty-four patients received treatment: allopurinol was used alone in 16 cases, allopurinol and miltefosine in 15 and allopurinol and N-methylglucamine antimoniate in 3. Twenty-seven (73\%) dogs were alive at the end of the study and 10 (27\%) had died. Mean survival time was 799.8 days (range 2-13320 days). Worsening of kidney disease was the most common cause of death.

In conclusion, the spectrum of clinical signs and laboratory abnormalities in the study group were similar to that reported in endemic areas. The therapeutic response was good considering the lack of licensed veterinary products in UK. Therefore, although CanL is an uncommon disease, practitioners should consider it especially in patients with compatible clinical signs and travel history.

\section{Mining clinical text notes to facilitate surveillance of Alabama Rot}

\section{Jenny Newman ${ }^{1,4}$, P-J.M. Noble ${ }^{1,2}$, Goran Nenadic ${ }^{4}$, Alan D. Radford ${ }^{3}$, David Walker ${ }^{5}$, Jessica Bacon², Philip H. Jones ${ }^{1}$}

\footnotetext{
Department of Epidemiology \& Population Health, University of Liverpool, Leahurst Campus, Neston, UK

School of Veterinary Science, University of Liverpool, Leahurst Campus, Neston, UK

Department of Infection Biology, University of Liverpool, Leahurst Campus, Neston, UK

School of Computer Science, University of Manchester, Manchester, UK Anderson Moores Veterinary Specialists, Winchester, UK
}

Between November 2012 and July 2014 there have been 44 confirmed cases of canine idiopathic cutaneous and renal glomerular vasculopathy (CRGV, "Alabama Rot") in the UK. The majority of these dogs died. The true prevalence is not known and as a new syndrome, it is likely that it is under-diagnosed.

We hypothesised that CRGV cases would have sentinel features in the text of the clinical record that might allow us to identify them using text-mining methods. If this were possible it would provide a clearer picture of the incidence of this disease in the UK veterinary population.

The veterinary notes of 33 animals with histologically confirmed disease formed the case group. A control group of 3300 dogs was selected at random from animals whose owners consulted their veterinary surgeon regarding an illness or injury during the period September 2012-January 2013, whose records were submitted to SAVSNET

Following cleaning of the consultation texts, clusters of words with similar meanings were identified. The likelihood of each cluster occurring within the text of confirmed cases, as compared to control, was calculated. Following removal of clusters likely to introduce or accentuate bias, those with an odds ratio in excess of 20 and $p<0.0001$ were identified, and these formed the key entity group. Clinical records were annotated to note the presence or absence of each entity. Annotated records were then modelled with an open source machine learning software workbench (WEKA). The clusters present in the model were sequentially adjusted to identify the optimum sensitivity and specificity achievable on this training dataset.

In this manner we were able to generate a model that recognised all 33 cases, and also identified 58 of the 3300 controls as fitting the same pattern of language. This provides the potential to feedback to clinicians that it may be appropriate to consider CRGV in their differential diagnosis. 